\title{
Attitudes Toward Depression Among Rehabilitation Participants With Acute Stroke: Evidence of an Age Cohort Effect
}

\author{
Patricia R. Roger and Doug Johnson-Greene \\ Johns Hopkins University School of Medicine
}

\begin{abstract}
Purpose/Objective: Depression is commonplace after acute stroke and is associated with increased morbidity and mortality. No data exist regarding attitudes about depression among older persons with acute stroke and their potential impact on self-report of depressive symptoms. The objective of this study was to determine if attitudes toward depression affect depression symptom reporting. Research Method/ Design: Cross-sectional using data from an inpatient rehabilitation unit. Seventy-two people with acute stroke were surveyed regarding their attitudes toward depression as part of a larger battery assessing their cognitive and emotional functioning. Results: Both age and cognitive status were significant predictors of attitudes toward depression. Older participants expressed significantly more negative attitudes about depression and seeking professional help compared with younger participants. Those with higher cognitive scores held more positive attitudes. However, attitudes about depression were unrelated to participants' responses on self-report measures of depression. Conclusions/Implications: Participants with stroke who were older were more likely to report negative attitudes about depressive symptoms than were younger participants. However, these attitudes do not appear to represent a barrier to their ability to accurately report the presence and severity of depressive symptoms.
\end{abstract}

Keywords: older persons, attitudes, depression, stroke, rehabilitation

Over a decade ago, depression in older adults was identified as a significant public health concern (NIH Consensus Development Panel on Depression in Late Life, 1992). Despite increased public awareness of this disease, the World Health Organization has recently reported that depression is the primary cause of disability in the world (Lopez, Mathers, Ezzati, Jamison, \& Murray, 2006). Late-life depression is associated with a wide range of medical comorbidities including cardiovascular, cerebrovascular, and musculoskeletal diseases (Charney et al., 2003), increased mortality (Gump, Matthews, Eberly, \& Chang, 2005; House, Knapp, Bamford, \& Vail, 2001), functional limitations (Pohjasvaara, Vataja, Leepavuori, Kaste, \& Erkinjuntti, 2001), and decreased quality of life (Zeiss, Lewinsohn, Rohde, \& Seeley, 1996). Higher rates of depression are also linked to an elevated rate of suicide among older adults (U.S. Public Health Service, 1999). Reported estimates of the prevalence of depressive symptoms among community-dwelling older adults range from $3 \%$ to $20 \%$ (Hybels \& Blazer, 2003). Among chronically ill older people, the rate has been approximately estimated to be $10 \%$, and for those in nursing homes, $16 \%$ to $30 \%$ (Hybels \& Blazer, 2003).

Understanding poststroke depression is critical for rehabilitation psychologists, given that over 700,000 people experience a stroke

Patricia R. Roger and Doug Johnson-Greene, Department of Physical Medicine and Rehabilitation, Division of Rehabilitation Psychology and Neuropsychology, Johns Hopkins University School of Medicine.

Correspondence concerning this article should be addressed to Patricia R. Roger, PhD, Department of Physical Medicine and Rehabilitation, Division of Rehabilitation Psychology and Neuropsychology, Johns Hopkins University School of Medicine, Good Samaritan POB Suite 406, 5601 Loch Raven Boulevard, Baltimore, MD 21239. E-mail: proger1@jhmi.edu each year in the United States and, as the National Institute of Neurological Disorders and Stroke noted, "approximately twothirds of these individuals survive and require rehabilitation" (National Institute of Neurological Disorders and Stroke, 2007, II 1). Depression is recognized as a common occurrence after stroke, with estimated prevalence rates ranging from $29 \%$ to $36 \%$ of all stroke survivors (Hackett, Yapa, Parag, \& Anderson, 2005). However, the underlying mechanisms and relationships between depressive symptoms and other clinical covariates remain unclear. For instance, after reviewing published studies examining the relationship between poststroke depression and other variables, Hackett and Anderson (2005) noted that existing models of poststroke depression are not sufficient to be clinically useful. Most significantly, "no reliable data are currently available to allow the easy identification of those patients at the greatest risk of experiencing depression at any time after the onset of stroke" (Hackett \& Anderson, 2005, p. 2301).

The diagnosis of depression and its subsequent treatment relies on an individual's self-report of depressed mood or symptoms. Therefore, it important to know if potential cohort effects exist and whether rehabilitation participants' attitudes toward depression affect their willingness to report depressive symptoms and openness to treatment. This is a particular concern with older adults, among whom depression may be underdiagnosed and undertreated (Gebretsadik, Jayaprabhu, \& Grossberg, 2006).

Ell (2006) noted that "older patients are less likely to voluntarily report depressive symptoms, may view depression as a moral weakness or character flaw, not an illness, and may be more likely to ascribe symptoms of depression to a physical illness" (p. 122). A cross-sectional study of 97 adults meeting Diagnostic and Statistical Manual of Mental Disorders (3rd ed., rev.) criteria for depression comparing self-reported depressive symptoms with ex- 
aminer-rated symptoms found that age was significantly negatively correlated with self-reported symptoms, as participants 60 years of age and older significantly underreported their depressive symptoms (Lyness et al., 1995).

It is important to note that as longevity increases, those age 65 years of age and older are becoming an ever more heterogeneous population. In the United States, there are now 34.7 million adults over the age of 65 years, according to the latest estimates. Of these, 12.6 million people are 75 to 84 years old and 3.8 million are 85 years or older (U.S. Census Bureau, 2005). Combining several generational cohorts and studying them as a whole may, in fact, mask differences within this population. It may be more useful for researchers and clinicians to examine the particularities of various subsets of older adults. For example, in a Swedish follow-up survey of stroke patients, over 11,000 stroke patients were asked, "Do you feel depressed?" 3 months after their stroke. Overall, $14.3 \%$ reported that they felt depressed, with patients younger than 65 years old significantly more likely to report depressed mood (Eriksson et al., 2004). It remains unclear, however, whether their responses reflect a difference in symptom frequency, differences in their willingness to endorse symptoms of depression, or both.

Older adults may be less likely to perceive major depression as a psychological or emotional problem (Hasin \& Link, 1988). In one study of 160 general hospital patients, individuals 65 years of age and older were more likely to say that they would approach no one for help with depression. They were also more likely to believe that older people are very unlikely to suffer from depression (Allen, Walker, Shergill, D'Ath, \& Katona, 1998). A 2006 study examining the attitudes of 332 nonacutely medically ill adults via a vignette based on the Geriatric Depression Screening Scale (Yesavage et al., 1983) compared the attitudes of those under and over 65 years of age. Older participants were significantly more likely than younger participants to hold negative attitudes about people with depression (Connery \& Davidson, 2006).

Given that some evidence suggests that attitudes about depression influence acknowledgement of depressive symptoms, treatment seeking, and treatment adherence, more information about the role of personal beliefs about mental illness, particularly depression, is necessary to reduce the barriers to effective treatment among rehabilitation populations, many of which are made up of older persons. Although attitudes are often conceptualized as predictors of outcomes, we chose attitudes as the outcome variable for this current study, because attitudes are thought to represent relatively stable beliefs and as such can be considered variables of interest in their own right. It is important to understand the correlates of attitudes toward depression, which may influence symptom reporting and, ultimately, rehabilitation outcomes (Chemerinski, Robinson, \& Kosier, 2001). Thus, our goal in this study was to explore the role of attitudes among a group shown to be at particularly high risk of developing depression: older adults with acute stroke. Our aim was to assess the relationship between attitudes toward depression, age, and endorsement of depressive symptoms. Consistent with existing research in this area, we hypothesized that more negative attitudes about depression would be found among older participants with acute stroke compared with younger participants, which would result in greater endorsement of depressive symptoms among younger participants with acute stroke compared with older ones.
Method

\section{Participants}

The study sample consisted of 72 men and women admitted to a hospital-based comprehensive inpatient rehabilitation program following an acute stroke. All participants were receiving rehabilitation psychology services as part of their routine clinical care. Recruitment occurred over a period of 12 months, during which consecutive admissions to the rehabilitation unit were considered for inclusion in the study. We included as participants those who were free from severe sensory (i.e., profound deafness, visual neglect, or blindness) or cognitive (i.e., delirium or history of dementia) impairments or significant aphasia that would impact comprehension or expression, as measured by a score of 2 on the Aphasia Severity Rating Scale from the Boston Diagnostic Aphasia Examination (Goodglass, 1983). All participants reported English as their primary language and had no history of alcohol or drug abuse or premorbid neurological illness. Participants who met inclusion criteria were provided with information about the study, and informed consent was obtained for those who agreed to participate. Although exact numbers are unavailable, only a very small number who were approached about involvement in the study refused to participate (approximately 15). This study was approved by the institutional review board of the medical institution.

The study sample had balanced representation in terms of age and gender. Just over half $(54 \% ; n=39)$ were women, and participants were between 43 and 89 years of age $(M=71$ years, $S D=10.2)$. The sample was predominantly Caucasian $(82 \% ; n=$ 59), and $15 \%$ of the participants were African American $(n=11$; 2 participants reported their racial or ethnic identification as "other"), which was consistent with the ethnic composition of the surrounding community. Forty-seven participants had incurred a right-side stroke, 22 had incurred a left-side stroke, and 3 were unspecified. Participants' mean Mini-Mental State Examination (MMSE; Folstein, Folstein, \& McHugh, 1975) score was 23.9 $(S D=4.0)$. Mean years of education was $12.0(S D=3.1)$. Functional Independence Measure (FIM) scores were available for 51 of the 72 participants in the study. The mean initial overall FIM score for those participants was $67.3(S D=14.4)$.

Given that premorbid experience of depression might be related to participants' poststroke depression as well as their attitudes toward mental illness, information regarding premorbid psychiatric illness was obtained for the majority of the sample $(67 \%)$. Thirty-eight $(53 \%)$ participants denied any premorbid psychiatric illness, and information was not available for 24 participants (33\%). Ten (14\%) participants reported a history of at least one major depressive episode, 1 participant reported a history of generalized anxiety disorder, and 1 reported a remote history of posttraumatic stress disorder. No significant differences were found using $t$ test comparisons of participants with premorbid depression versus those without a premorbid history of depression in attitudes toward depression, self-rated depression, or scores on standardized depression measures.

\section{Measures}

As part of larger study of poststroke functioning and quality of life, several cognitive and emotional assessments were adminis- 
tered. Basic cognitive functioning was estimated using the MMSE. Participants were administered two self-report measures of depression in random order: the frequently used Center for Epidemiologic Studies Depression Scale (CES-D; Radloff, 1977) and the Stroke Inpatient Depression Inventory (SIDI; Rybarczyk, Winemiller, Lazarus, \& Haut, 1996), a depression measure specifically validated for use with this population. Participants were also asked to rate their current mood on a 10-point Likert-type scale, with 1 representing the absence of depression and 10 representing severe depression (depression rating).

Additionally, participants completed a brief questionnaire designed to assess their attitudes about depression. Using a 5-point Likert-type scale (from $1=$ strongly disagree to $5=$ strongly agree), participants rated the extent to which they agreed with the following three statements: (a) "When people feel downhearted and blue, they should keep it to themselves"; (b) "People who are depressed should solve their problems on their own"; and (c) "Depression is a sign of weakness or personal failure." Items were summed to create a depression attitudes score that ranged from 3 to 15 , with high scores representing more negative attitudes toward depression. Cronbach's alpha of interitem reliability for the three items was .65 , suggesting an acceptable level of internal consistency for a new, exploratory measure.

\section{Statistical Analyses}

Means, standard deviations, and Pearson correlations between measures were calculated. A stepwise linear regression with the entire sample was performed to identify the variables' independent contribution to attitudes toward depression. The sample was then divided using the median age of 73 years as a cut point $(n<73=$ $35 ; n \geq 73=37$ ). Independent sample $t$ tests were used to compare younger and older cohorts on variables of interest.

\section{Results}

Participants' self-reported depression ratings ranged from 1 to 10 , with a mean of $3.9(S D=3.0)$. On the depression attitudes questionnaire, scores for each of the three items ranged from 1 to 5 and total scores ranged from 3 to 13 , indicating that participants' responses ranged along the entire continuum of possible responses for attitudes about depression.

Comparisons between the older and younger age cohorts using independent sample $t$ tests showed no group differences for race, gender, side of stroke, years of education, cognitive functioning as measured by MMSE scores, initial FIM score, or self-reported depression ratings between older and younger participants. However, a significant difference between the two groups was found in their attitudes toward depression, with older participants endorsing attitudes that were more negative than those endorsed by younger participants, $t(70)=2.7, p<.01$. A summary of these findings can be found in Table 1 .

As expected, the two measures of depression were significantly correlated with one another, with a Pearson coefficient of $.42(p<$ .01). Likewise, participants' self-rating of their level of depression correlated $.38(p<.01)$ with their score on the SIDI and $.44(p<$ $.01)$ with their score on the CES-D. FIM scores were inversely correlated with scores on the SIDI $(r=-.32, p<.05)$, although
Table 1

Comparison of Younger and Older Participants With Acute Stroke

\begin{tabular}{lccccr}
\hline \multicolumn{1}{c}{ Measure } & $\begin{array}{c}\text { Old } \\
(\geq 73 \text { years })\end{array}$ & $\begin{array}{c}\text { Young } \\
(<73 \text { years })\end{array}$ & $\begin{array}{c}\text { Total } \\
\text { sample }\end{array}$ & $n$ & $t$ \\
\hline$n$ & 37 & 35 & 72 & & \\
MMSE & $23.0 \pm 4.3$ & $24.8 \pm 3.4$ & $23.9 \pm 4.0$ & 71 & -1.9 \\
$\begin{array}{l}\text { FIM } \\
\text { Attitudes toward }\end{array}$ & $65.0 \pm 13.7$ & $69.9 \pm 14.9$ & $67.3 \pm 14.4$ & 51 & -1.2 \\
$\quad \begin{array}{l}\text { depression } \\
\text { Depression }\end{array}$ & $8.1 \pm 3.0$ & $6.3 \pm 2.5$ & $7.2 \pm 2.9$ & & $2.7^{*}$ \\
$\quad$ self-rating & $4.0 \pm 3.0$ & $4.0 \pm 3.0$ & $3.9 \pm 3.0$ & & 0.2 \\
$\begin{array}{l}\text { SIDI } \\
\text { CES-D }\end{array}$ & $11.1 \pm 6.4$ & $9.3 \pm 5.4$ & $10.3 \pm 5.9$ & 69 & 1.1 \\
\hline
\end{tabular}

Note. Higher attitude scores indicate greater negative attitudes toward depression. MMSE = Mini-Mental State Exam; FIM = Functional Independence Measure; SIDI = Stroke Inpatient Depression Inventory; CES$\mathrm{D}=$ Center for Epidemiologic Studies Depression Scale. ${ }^{*} p<.01$.

they did not correlate with scores on the other two measures of depression.

Attitudes toward depression total score was significantly correlated with age $(r=.37, p<.01)$, with older participants reporting more negative attitudes toward depression than younger participants. However, age itself was not significantly correlated with any of our depression measures. In contrast, participants' attitudes toward depression were not correlated with their scores on any measures of depression (see Table 2). MMSE scores were inversely correlated with attitudes toward depression $(r=-.31, p<$ .01 ), suggesting that the more cognitively impaired participants endorsed more negative attitudes toward depression, compared with the less cognitively impaired participants.

To determine clinical predictors of self-reported depression and attitude scores, we computed a stepwise regression analysis to determine which variables accounted for the greatest variance in depression attitudes. First, we predicted depression attitude scores using age, MMSE scores, and scores for the two standardized depression instruments as predictors. To control for stroke severity, we used participants' initial FIM score as a covariate, but its inclusion did not significantly affect the results. Similarly, an alternate equation was modeled, with participants' premorbid history of depression entered as a covariate, but it was not a significant predictor. Measures of depression were shown to be unrelated to depression attitude scores and so were not included in the final model. Age and cognitive status accounted for $14 \%$ and $5 \%$ of the significant variance in attitudes toward depression, respectively (see Table 3 ).

\section{Discussion}

Consistent with our first hypothesis, we found a statistically significant difference in attitudes toward depression among older and younger study participants with acute stroke. Older participants were more likely than younger participants to agree with statements indicating that depression is a personal failure that does not require treatment. Stroke survivors younger than 73 years were more likely to indicate a more tolerant attitude about depression 
and presumably greater openness to treatment compared with the older cohort.

Because of their apparent differences in attitudes toward depression, our second hypothesis predicted that the two age groups would have different rates for endorsing depressive symptoms. However, contrary to our hypothesis, the two groups did not differ in their endorsement of depressive symptoms identified with objective depression measures and a self-reported depression rating. In contrast to previous studies in which lower rates of depression found among the older people were thought to reflect underreporting of symptoms (Connery \& Davidson, 2006; Lyness et al., 1995), no relationship was found between participants' attitudes toward depression and their responses on several self-report measures of depression. This relationship did not change after controlling for age, cognitive functioning, and initial functional impairment.

A strength of this study is the use of a racially representative and gender-balanced sample. Its generalizability is limited by the fact that the participants were acute stroke survivors who were able to participate in rehabilitation. Additionally, the majority of participants had experienced right-sided strokes. Because rehabilitation consumers with left-sided strokes were frequently excluded because of language impairments, the results of this study may not be representative of those individuals. Although no differences in mean MMSE scores were found between the older and younger cohorts, it is possible that cognitive limitations may have affected participants' responses to items. MMSE scores were inversely related to participants' attitudes, as participants with lower MMSE scores endorsed more negative attitudes toward depression. However, our sample's mean MMSE score is consistent with the ageand education-corrected cutoff score of 24 for 70- to 74-year-olds and 22 for 75- to 79-year-olds suggested by Iverson's (1998) community-dwelling population norms, so we have reason to believe that participants in the study performed similarly to their same-age peers without stroke.

The frequency of depression after stroke has led to speculation that it is a normal reaction to the cognitive and physical limitations stroke survivors often experience. As such, their depressive symptoms may be overlooked or downplayed by family and rehabilitation team members or by stroke survivors themselves. In addition, some older individuals may harbor negative attitudes toward the experience of depression, as our findings suggest. For some stroke

Table 2

Pearson Correlation Coefficients

\begin{tabular}{lcccccc}
\multicolumn{1}{c}{ Variable } & 1 & 2 & 3 & 4 & 5 & 6 \\
\hline Age & $-.31^{\dagger}$ & -.14 & $.37^{\dagger}$ & .05 & .17 & .12 \\
1. MMSE & - & $.44^{\dagger}$ & $-.31^{\dagger}$ & -.15 & -.08 & -.10 \\
2. Initial FIM & & - & -.17 & -.06 & $-.32^{*}$ & -.26 \\
3. Attitudes toward & & & & & & \\
$\quad$ depression & & & - & -.18 & .04 & .05 \\
4. Depression self-ratings & & & & - & $.38^{\dagger}$ & $.44^{\dagger}$ \\
5. SIDI & & & & & - & $.42^{\dagger}$ \\
6. CES-D & & & & & & - \\
\hline
\end{tabular}

Note. $\quad$ MMSE $=$ Mini-Mental State Exam; FIM $=$ Functional Independence Measure; SIDI = Stroke Inpatient Depression Inventory; CES-D = Center for Epidemiologic Studies Depression Scale.

${ }^{\dagger} p<.01$, two-tailed. ${ }^{*} p<.05$, two-tailed.
Table 3

Summary of Regression Analysis for Variables Predicting Attitudes Toward Depression $(N=72)$

\begin{tabular}{lccccc}
\hline Variable & $R$ & Adjusted $R^{2}$ & $\Delta R^{2}$ & $F$ & $\beta$ \\
\hline Age & .37 & .14 & .14 & $10.9^{\dagger}$ & $.38^{\dagger}$ \\
MMSE & .44 & .20 & .05 & $4.1^{*}$ & $-.24^{*}$ \\
\hline
\end{tabular}

Note. $\mathrm{MMSE}=$ Mini-Mental State Exam.

${ }^{\dagger} p<.01 .{ }^{*} p<.05$.

survivors, these attitudes may present internal barriers that prevent them from seeking help for depression and increase their risk of depression-related poststroke morbidity. However, our findings indicate that self-reported and standardized measures of depression may nonetheless be useful in identifying older people who are experiencing depressive symptoms. Our study found that despite a tendency for older participants to express negative attitudes toward seeking or receiving professional help for depression, participants' willingness to endorse depressive symptoms on self-report depression measures appears to remain independent of these attitudes and should be considered a legitimate source of information about the presence and severity of depressive symptoms. However, the extent to which attitudes about depression may influence willingness to seek and accept treatment for depression and to what extent they may be barriers to participation in rehabilitation programs remains unclear. In future studies, researchers should explore the impact of depression attitudes on treatment in other rehabilitation populations and whether attitudes toward depression change longitudinally as the process of rehabilitation continues.

\section{References}

Allen, R. L., Walker, Z., Shergill, S. S., D’Ath, P., \& Katona, C. L. E. (1998). Attitudes to depression in hospital inpatients: A comparison between older and younger subjects. Aging and Mental Health, 2, 36-39.

Charney, D. S., Reynolds, C. F., III, Lewis, L., Lebowitz, B. D., Sunderland, T., Alexopoulos, G. S., et al. (2003). Depression and bipolar support alliance consensus statement on the unmet needs in diagnosis and treatment of mood disorders in late life. Archives of General Psychiatry, 60, 664-672.

Chemerinski, E., Robinson, R. G., \& Kosier, J. T. (2001). Improved recovery in activities of daily living associated with remission of poststroke depression. Stroke, 32, 113-117.

Connery, H., \& Davidson, K. M. (2006). A survey of attitudes to depression in the general public: A comparison of age and gender differences. Journal of Mental Health, 15, 179-189.

Ell, K. (2006). Depression care for the elderly: Reducing barriers to evidence-based practice. Home Health Care Services Quarterly, 25, $115-148$.

Eriksson, M., Asplund, K., Glader, E.-L., Norrving, B., Stegmayr, B. Terent, A., et al. (2004). Self-reported depression and use of antidepressants after stroke: A national survey. Stroke, 35, 936-941.

Folstein, M. F., Folstein, S. E., \& McHugh, P. R. (1975). "Mini-mental state": A practical method for grading the cognitive state of patients for the clinician. Journal of Psychiatric Research, 12, 189-198.

Gebretsadik, M., Jayaprabhu, S., \& Grossberg, G. T. (2006). Mood disorders in the elderly. Medical Clinics of North America, 90, 789-805.

Goodglass, H. (with Kaplan, E.). (1983). The assessment of aphasia and related disorders (2nd ed.). Media, PA: Williams \& Wilkins. 
Gump, B. B., Matthews, K. A., Eberly, L. E., \& Chang, Y. (2005). Depressive symptoms and mortality in men: Results from the Multiple Risk Factor Intervention Trial. Stroke, 36, 98-102.

Hackett, M. L., \& Anderson, C. S. (2005). Predictors of depression after stroke: A systematic review of observational studies. Stroke, 36, 22962301.

Hackett, M. L., Yapa, C., Parag, V., \& Anderson, C. S. (2005). Frequency of depression after stroke: A systematic review of observational studies. Stroke, 36, 1330-1340.

Hasin, D., \& Link, B. (1988). Age and recognition of depression: Implications for a cohort effect in major depression. Psychological Medicine, $18,683-688$.

House, A., Knapp, P., Bamford, J., \& Vail, A. (2001). Mortality at 12 and 24 months after stroke may be associated with depressive symptoms at 1 month. Stroke, 32, 696-701.

Hybels, C. F., \& Blazer, D. G. (2003). Epidemiology of late-life mental disorders. Clinics in Geriatric Medicine, 19, 663-696.

Iverson, G. (1998). Interpretation of Mini-Mental State Examination scores in community-dwelling elderly and geriatric neuropsychiatry patients. International Journal of Geriatric Psychiatry, 13, 661-666.

Lopez, D. A., Mathers, C. D., Ezzati, M., Jamison, D. T., \& Murray, C. J. L. (2006). Measuring the global burden of disease and risk factors, 1990-2001. In D. A. Lopez, C. D. Mathers, M. Ezzati, D. T. Jamison, \& C. J. L. Murray (Eds.), Global burden of disease and risk factors (pp. 1-13). Washington, DC: World Bank. Retrieved February 29, 2008, from http://files.dcp2.org/pdf/GBD/GBD.pdf

Lyness, J. M., Cox, C., Curry, J., Conwell, Y., King, D. A., \& Caine, E. D. (1995). Older age and the underreporting of depressive symptoms. Journal of the American Geriatrics Society, 43, 216-221.

National Institute of Neurological Disorders and Stroke. (2007, November 1). Post-stroke rehabilitation fact sheet. Retrieved November 2, 2007, from http://www.ninds.nih.gov/disorders/stroke/poststrokerehab.htm
NIH Consensus Development Panel on Depression in Late Life. (1992) NIH consensus conference: Diagnosis and treatment of depression in late life. Journal of the American Medical Association, 268, 1018-1024.

Pohjasvaara, T., Vataja, R., Leepavuori, A., Kaste, M., \& Erkinjuntti, T. (2001). Depression is an independent predictor of poor long-term functional outcome after stroke. European Journal of Neurology, 8, 315319.

Radloff, L. S. (1977). The CES-D Scale: A self-report depression scale for research in the general population. Applied Psychological Measurement, 1, 385-401.

Rybarczyk, B., Winemiller, D. R., Lazarus, L. W., \& Haut, A. (1996). Validation of a depression screening measure for stroke inpatients. American Journal of Geriatric Psychiatry, 4, 131-139.

U.S. Census Bureau. (2005). United States General Data Characteristics. 2005. Retrieved November 22, 2006, from http://factfinder.census.gov/

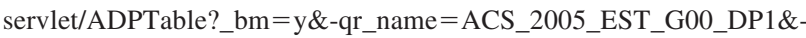
geo_id=01000US\&-gc_url=null $\&$-ds_name $=$ ACS_2005_EST_G00

U.S. Public Health Service. (1999). The Surgeon General's call to action to prevent suicide. Washington, DC: Author.

Yesavage, J. A., Brink, T. L., Rose, T. L., Lum, O., Huang, V., Adey, M. B., \& Leirer, V. O. (1983). Development and validation of a geriatric depression screening scale: A preliminary report. Journal of Psychiatric Research, 17, 37-49.

Zeiss, A. M., Lewinsohn, P. M., Rohde, P., \& Seeley, J. R. (1996) Relationship of physical disease and functional impairment to depression in older people. Psychology and Aging, 11, 572-581.

Received June 29, 2007

Revision received November 26, 2007 Accepted December 19, 2007 